\title{
O I 25 Impact of efavirenz and nevirapine on pharmacokinetics of lopinavir/ritonavir as tablets and capsules in African patients
} C Kityo*1, AS Walker ${ }^{2}$, F Lutwana ${ }^{3}$, F Ssali ${ }^{4}$, R Nalumenya ${ }^{3}$, D Tumukunde ${ }^{4}$, J Kawiya ${ }^{5}$, P Munderi ${ }^{6}$, A Reid7, CF Gilks ${ }^{8}$, DM Gibb² and SH Khoo ${ }^{9}$

Address: ${ }^{1} \mathrm{CRC}$, Uganda, Uganda, ${ }^{2} \mathrm{MRC}$ Clinical Trials Unit, London, UK, ${ }^{3}$ Infectious Diseases Institute, Mulago, Uganda, ${ }^{4}$ Joint Clinical Trials Unit, London, UK, ${ }^{5}$ Joint Clinical Research Centre, Kampala, Uganda, ${ }^{6} \mathrm{MRC} /$ UVRI Research Unit on AIDS, Entebbe, Uganda, ${ }^{7}$ University of Zimbabwe, Harare, Zimbabwe, ${ }^{8}$ Imperial College, London, UK and ${ }^{9}$ Liverpool University, Liverpool, UK

* Corresponding author

from Ninth International Congress on Drug Therapy in HIV Infection

Glasgow, UK. 9-13 November 2008

Published: 10 November 2008

Journal of the International AIDS Society 2008, I I (SuppI I):OI0 doi:I0.1 I86/I758-2652-II-SI-OIO

This abstract is available from: http://www.jiasociety.org/content/II/SI/OIO

(c) 2008 Kityo et al; licensee BioMed Central Ltd.

\section{Purpose of the study}

With NNRTI, the recommended dose of lopinavir/r (LPV/ r) is 4 capsules $(533 / 133 \mathrm{mg}) \mathrm{BD}$. With LPV/r tablets, the closest doses are 2 tablets (400/100 mg) BD or 3 tablets [tabs] (600/150 mg) BD. Improved bioavailability of the tablet suggests that 2 tabs BD should be sufficient, but PK data are few and generally from Caucasians.

\section{Methods}

We conducted a three-period crossover PK study in 40 patients receiving $\mathrm{LPV} / \mathrm{r}$ tablets with NNRTIs (21 EFV,19 NVP) second-line (first PI) in one Ugandan centre in the DART trial. Patients on 3 tabs BD underwent 6-point PK sampling $(0,2,4,6,8$ and 12 hours) after observed intake with a standardised breakfast. They then switched to 4 caps BD for 2 weeks before a second PK, then switched to 2 tabs BD for 2 weeks before a third PK, after which they returned to 3 tabs BD. Analysis excluded two patients with minimal LPV/r exposure on 4 caps BD.

\section{Summary of results}

6/20 EFV and 13/18 NVP patients included were female (reflecting contra-indication of EFV in women with childbearing potential), with median age 41 and 35 years, and weight 60 and $64 \mathrm{~kg}$, respectively. On EFV, mean (SD) LPV AUC was 104 (54), 70 (27) and 63 (33) ug.h/l on 3 tabs $\mathrm{BD}, 4$ caps $\mathrm{BD}$, and 2 tabs $\mathrm{BD}$, respectively, with corresponding GMR vs. 4 caps BD 1.40 (90\% CI 1.18-1.65, $\mathrm{p}=0.002)$ and $0.82(0.68-0.99, \mathrm{p}=0.09)$. On NVP, mean
(SD) LPV AUC was 118 (33), 77 (39) and 69 (34) ug.h/l, respectively, with corresponding GMR vs. 4 caps BD 1.66 $(1.46-1.88, \mathrm{p}<0.001)$ and $0.90(0.77-1.06, \mathrm{p}=0.27)$ for 3 and 2 tabs BD, respectively. On EFV, 15\%/15\%/40\% had $\mathrm{C} 12$ (trough) $<1 \mathrm{ug} / \mathrm{ml}(\mathrm{p}=0.1)$ with corresponding GMR vs. 4 caps BD $1.48(1.09-2.02, \mathrm{p}=0.04)$ and 0.62 $(0.39-0.98, \mathrm{p}=0.08)$ for 3 and 2 tabs $\mathrm{BD}$, respectively. On NVP, $0 \% / 22 \% / 28 \%$ had $\mathrm{C} 12<1 \mathrm{ug} / \mathrm{ml}(\mathrm{p}=0.06)$ with corresponding GMR vs. 4 caps BD 2.31 (1.64-3.24, p < $0.001)$ and $0.80(0.57-1.12, \mathrm{p}=0.26)$ for 3 and 2 tabs $\mathrm{BD}$, respectively. However, $40 \% / 5 \% / 15 \% \mathrm{EFV}$ and $56 \% / 28 \% /$ $17 \%$ NVP patients had $\mathrm{C} 12>5 \mathrm{ug} / \mathrm{ml}$. There was no effect of sex, age, weight or BMI on AUC or C12.

\section{Conclusion}

When co-administered with NVP or EFV in African patients, LPV AUC and C12 are higher with 3 tablets BD and lower with 2 tablets BD compared to 4 capsules BD. Higher plasma levels on 3 tablets BD may lead to greater long-term toxicity, whereas low plasma C12 on 2 tablets $\mathrm{BD}$ may increase the risk of virological failure. However, AUC on 4 caps BD was lower than expected, and tablet AUC variability was higher than expected. Further PK sampling is therefore ongoing to estimate PK parameters in African patients taking $2 \mathrm{LPV} / \mathrm{r}$ tablets $\mathrm{BD}$ without NNRTIs. 